\title{
NanoMi Open Source (S)TEM Platform: Initial SEM Implementation.
}

Marek Malac ${ }^{1}$, Karan Kumar ${ }^{2}$, Darren Wen ${ }^{3}$, Jesus Alejandro Marin-Calzada ${ }^{4}$, Martin Cloutier ${ }^{5}$, Mark Salomons $^{6}$, Darren Homeniuk ${ }^{5}$, Sean Chen ${ }^{7}$, Jason Pitters ${ }^{5}$, Doug Vick ${ }^{5}$, Drew Price ${ }^{5}$, Misa Hayashida ${ }^{8}$ and Ray Egerton ${ }^{4}$

${ }^{1}$ National Research of Council, Alberta, Canada, ${ }^{2}$ University of Alberta, United States, ${ }^{3}$ Dept. of Integrated Engineering, University of British Columbia,V6T 1Z4, Vancouver, Canada., United States, ${ }^{4}$ Department of Physics, University of Alberta, T6G 2E1, Edmonton, Canada., United States, ${ }^{5}$ NRCNANO, 11421 Saskatchewan Drive, T6G 2M9, Edmonton, Canada., United States, ${ }^{6}$ NRC-NANO, 11421 Saskatchewan Drive, T6G 2M9, Edmonton, Canada., Edmonton, Alberta, Canada, ${ }^{7}$ Department of Physics \& Astronomy, 325 - 6224 Agricultural Rd., Vancouver, Canada., United States, ${ }^{8}$ National Research of Council, Edmonton, Alberta, Canada

We provide an update on a public-license electron microscope (EM) platform [1], referred to as NanoMi. The optics are modular, allowing assembly of a TEM, scanning TEM (STEM) or an SEM (discussed here) within an independent vacuum envelope.

NanoMi uses electrostatic lenses that are amenable to future ion-beam development and eliminate the need for water cooling [2]. Elements of the control software and modeling tools are released under GPL3 [3] license and can be found at [4]. Hardware blueprints are intended for release under CERN Weakly Reciprocal Open Hardware License v.2 [2]. To ensure NanoMi module interchangeability, a description of their electrical, mechanical and optical interfaces is being developed.

A NanoMi SEM utilizes the three-lens system optics as shown in Fig. 1. Focal lengths agree with the expected excitation-voltage ratio $U_{\mathrm{R}}[2]$ and with finite element simulations. Fig. 2 shows the cross section of the column with SEM optics. Fig. 3 shows an Einzel lens cross section [2].

Using a tungsten hairpin source, a small probe is obtained after demagnification by about $1,000 x$. The field of view is can be as wide as $200 \mu \mathrm{m}$, although image distortion is currently severe at the outer limits. A single set of deflector plates was used to scan the beam, introducing beam tilt up to $10 \mathrm{mrad}$. The deflector produces up to $\sim 3.8 \times 10^{4} \mathrm{~V} / \mathrm{m}$ over $\sim 10 \mathrm{~mm}$ of the beam path. A probe stigmator has not been implemented at this time. Each lens is biased from own high voltage (HV) power supply but a voltage divider utilizing a single HV supply for all lenses is being developed. NanoMi provides sufficient precision in lens placement and centering to obtain images without beam shift and tilt adjustments between lenses, even if the construction uses an ordinary machine shop.

An Everhart-Thornley (ET) detector was used to collect secondary-electron signal. The column dimensions and optics allow for straightforward implementation of scanning transmission electron microscopy (STEM) by placing a detector beyond an electron-transparent sample. At present, the resolution is limited by noise and $60 \mathrm{~Hz}$ pickup, which we are working to eliminate. 


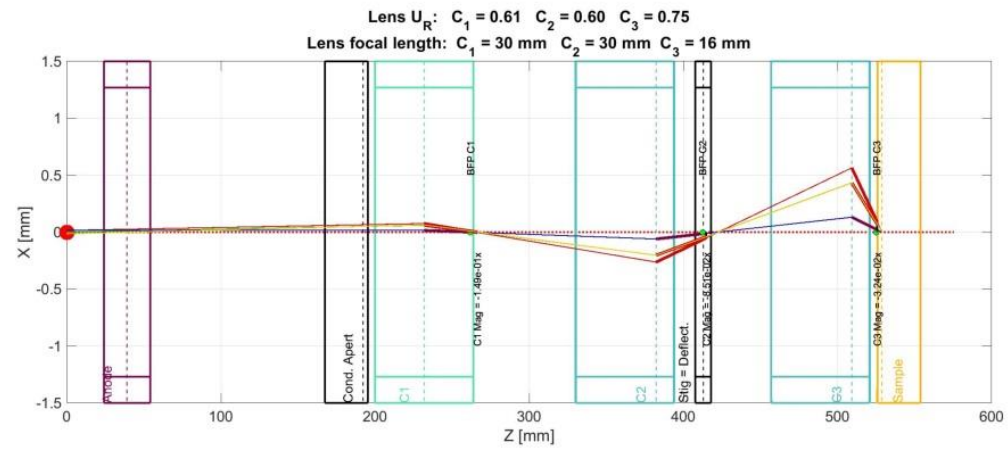

Fig. 1. The NanoMi SEM utilizes three Einzel lens optics. The focal lengths are $\mathrm{C} 1=\mathrm{C} 2=30 \mathrm{~mm}, \mathrm{C} 3 \approx 16 \mathrm{~mm}$ is used for probe focusing on the sample.
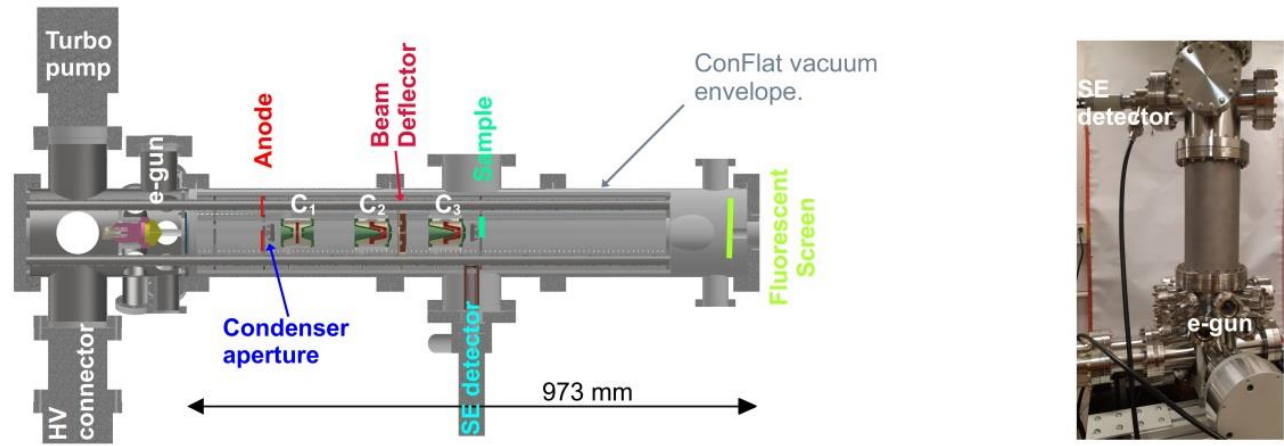

Fig. 2 Cross section of the column indicating the location of the Einze lens in the 6" diameter ConFlat vacuum hardware. Gun is located on the left, sample is indicated by an arrow and a florescent screen is on the right. The optical path is $973 \mathrm{~mm}$ from gun to the screen. The microscope is oriented vertically with the gun located at the bottom, see right panel. Here $C_{1}$ is an symmetric Einzel lens [1].

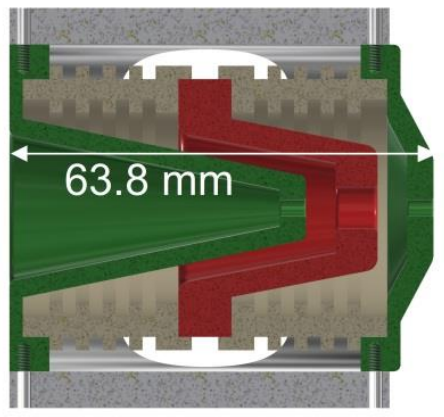

Fig. 3 Cross section of asymmetric Einzel lens used for $\mathrm{C}_{2}$ and $\mathrm{C}_{3}$ of the SEM column. Grounded electrodes are depicted in green and biased central electrode in red. They are separated by a PEEK insulator in brown. The gray outer shell is grounded.

\section{References}

[1] M. Malac et. al. Micr. and Microanal. 26 (S2) (2020), p. 1810-1811.

[2] G. Rempfer, J. Appl. Phys. 57 (1985), p. 2385.

[3] https://www.gnu.org/licenses/gpl-3.0.en.html and https://opensource.org/CERN-OHL-W [4] nanomi.org and https://osf.io/bpj73, DOI: 10.17605/OSF.IO/BPJ73 